\title{
Breit corrections to individual atomic and molecular orbital energies
}

\author{
Karol Kozioł, ${ }^{1, a)}$ Carlos A. Giménez, ${ }^{1,2}$ and Gustavo A. Aucar ${ }^{1,2, b)}$ \\ ${ }^{1}$ Institute for Modelling and Innovative Technology, IMIT, Corrientes, Argentina \\ ${ }^{2}$ Physics Department, Natural and Exact Science Faculty, Northeastern University of Argentina, \\ Corrientes, Argentina
}

(Received 1 December 2017; accepted 11 January 2018; published online 29 January 2018)

\begin{abstract}
Several issues concerning Breit correction to electron-electron interaction in many-electron systems, which are important in precise atomic and molecular calculations, are presented. At first, perturbative versus self-consistent calculations of Breit correction were studied in selected cases. Second, the Zdependence of Breit contribution per subshell is shown, based on values calculated for selected atoms with $30 \leq Z \leq 118$. Third, the relations between magnetic and retardation parts of Breit interaction are analyzed. Finally, Gaunt contribution calculated for $\mathrm{Kr}, \mathrm{Xe}$, and $\mathrm{Rn}$ noble gas atoms and its isoelectronic $\mathrm{HBr}, \mathrm{HI}$, and HAt diatomic molecules has been compared to full-Breit atomic calculations. We found that Breit corrections should be treated by self-consistent calculations and that there is a functional dependence of those corrections for subshells as $\varepsilon_{n l}^{B r e i t}(Z) \simeq a \times Z^{b}$. We also found that molecular Gaunt corrections are close to their atomic counterparts for inner electrons though they are not for outer orbitals. In any case, accurate calculations must include retardation correction in addition to Gaunt. Published by AIP Publishing. https://doi.org/10.1063/1.5017986
\end{abstract}

\section{INTRODUCTION}

Calculations of both atomic structure and properties for the medium to high atomic number range require careful consideration of relativistic effects. ${ }^{1}$ The Breit interaction gives the leading relativistic correction to the instantaneous Coulomb electron-electron interaction potential in quantum electrodynamics. Its inclusion is required for precision calculations of binding energies and transition rates in heavy atoms. $^{2}$

There has been an intensive discussion in the past years on whether the two-electron Breit interaction terms should be used perturbatively or variationally. ${ }^{3-7} \mathrm{Breit}^{8-10}$ and later Bethe and Salpeter ${ }^{3}$ pointed out that the Breit operator should only be treated as a first-order perturbation because its inclusion in an unperturbed Hamiltonian would led to a result inconsistent with quantum electrodynamics. On the other hand, more recently Quiney et $a l^{6}{ }^{6}$ and Ishikawa et al. ${ }^{11,12}$ have shown that the correct inclusion of the Breit term in the self-consistent-field procedure does not lead to "variational collapse"13 or to a result inconsistent with quantum electrodynamics. In line with this, Grant et al. ${ }^{14,15}$ pointed out that a variational procedure for Breit terms is preferred in a subsequent treatment of electron correlation. Moreover, Lindroth et al. ${ }^{16}$ stated that a variational self-consistent treatment of the Breit interaction is very important for properties of orbitals, especially for the valence ones, in many-electron systems.

On the other hand, Chen et al. ${ }^{17}$ and further Costa et al. ${ }^{18}$ reported that the $K^{h} \alpha_{1} / K^{h} \alpha_{2}$ line intensity ratio is sensitive to the inclusion of the Breit interaction. They found that for

a)Electronic mail: mail@karol-koziol.net

b)Electronic mail: gaa@unne.edu.ar low- $Z$ elements the inclusion of the Breit term decreases the $K^{h} \alpha_{1} / K^{h} \alpha_{2}$ ratio by $48 \%(Z=12)$ to $20 \%(Z=20)$. For medium- and high- $Z$ atoms $(Z \gtrsim 30)$, the effect is reversed, meaning that including the Breit term increases the $K^{h} \alpha_{1} / K^{h} \alpha_{2}$ ratio by about $5 \%-7 \%$. Pernpointner ${ }^{19,20}$ showed that Breit-type integrals at the SCF level exhibit a weak but noticeable effect for molecular properties, such as electric field gradient and ionization spectrum, of $\mathrm{TlH}$. Further studies of Quiney et al. ${ }^{21}$ focused on the effect of Breit corrections to the potential energy surface and vibration-rotation levels of water. Beside, Thierfelder and Schwerdtfeger pointed out ${ }^{22}$ that the variational treatment of the Breit interaction achieves Koopmans theorem.

Recently, Chantler et $a l .{ }^{23}$ studied the convergence of the Breit interaction in self-consistent and configurationinteraction approaches. The $Z$-dependence of Breit contribution to the total energy of the ground state of selected atoms with $2 \leq Z \leq 102$ has been studied by Mann and Johnson, ${ }^{24}$ and $Z^{3.6}$ dependence has been found. They also studied the relative importance of magnetic (Gaunt) and retardation parts of the Breit interaction and found that the energy shift due to retardation is about $10 \%$ of the Gaunt energy shift for ground states of atoms in the range $Z=2-Z=102$, and that the Gaunt and the retardation contributions are of opposite signs. In the extensive study of Huang et al. ${ }^{25}$ the Breit contributions to the orbital binding energies for all atoms with $2 \leq Z \leq 106$ have been presented. Niskanen $e t a l .{ }^{26}$ found that the Gaunt contribution to the single and double $1 s$ electron ionization energies of noble gases scales approximately as $Z^{3.3}$.

Including the Breit interaction term in the self-consistentfield procedure using the Gaussian basis set, applying to general-purpose molecular calculations, was established well last years. ${ }^{29-31}$ However these studies implement only the dominant magnetic part of the Breit interaction in calculations. 
In this work, we show new insights about several issues concerning Breit correction, which are important in precise atomic and molecular calculations. At first, results of perturbative and self-consistent calculations of Breit correction are analyzed in selected atomic systems. Second, the Zdependence of Breit contribution per subshell is shown, based on values calculated for selected atoms with $30 \leq Z \leq 118$. Third, the relations between magnetic and retardation parts of Breit interaction have been studied. Finally, Gaunt contributions calculated for $\mathrm{Kr}, \mathrm{Xe}$, and $\mathrm{Rn}$ noble gas atoms and its iso-electronic $\mathrm{HBr}$, $\mathrm{HI}$, and $\mathrm{HAt}$ diatomic molecules are compared with full-Breit atomic calculations. We want to give new insights on the behavior of Breit and Gaunt corrections to the atomic or molecular orbital energies of atoms and diatomic molecules.

\section{THEORETICAL BACKGROUND}

\section{A. MCDF methods}

The methodology of Multi-Configuration Dirac-HartreeFock (MCDF) calculations performed in the present studies is similar to the one published earlier, in several papers (see, e.g., Refs. 32-37). The effective Hamiltonian for an $N$-electron system is expressed by

$$
\hat{H}=\sum_{i=1}^{N} \hat{h}_{D}(i)+\sum_{j>i=1}^{N} V_{i j}
$$

where $\hat{h}_{D}(i)$ is the Dirac one-particle operator for the $i$ th electron and the terms $\hat{V}_{i j}$ account for the effective electron- electron interactions and depend on the photon propagator. When considering the virtual one-photon exchange, it may be expressed in any of the two gauges, the Feynman (Lorentz) gauge or the Coulomb gauge. They do have different actual expressions that need to be clarified because they will be used in this work. ${ }^{38}$

The interaction potential in the Feynman gauge (also called the Møller interaction ${ }^{39}$ ) is

$$
\hat{V}_{i j(F)}=\left(1-\boldsymbol{\alpha}_{i} \cdot \boldsymbol{\alpha}_{j}\right) \frac{e^{i \omega_{i j} r_{i j}}}{r_{i j}},
$$

and in the Coulomb gauge it is

$$
\hat{V}_{i j(C)}=\left[\frac{1}{r_{i j}}-\boldsymbol{\alpha}_{i} \cdot \boldsymbol{\alpha}_{j} \frac{e^{i \omega_{i j} r_{i j}}}{r_{i j}}-\left(\boldsymbol{\alpha}_{i} \cdot \nabla_{i}\right)\left(\boldsymbol{\alpha}_{j} \cdot \nabla_{j}\right) \frac{e^{i \omega_{i j} r_{i j}}-1}{\omega_{i j}^{2} r_{i j}}\right] .
$$

The latter is a sum of the Coulomb interaction $\hat{V}_{i j}^{C}$ operator and the transverse Breit $\hat{V}_{i j}^{B}$ operator, ${ }^{8-10}$

$$
\hat{V}_{i j}=\hat{V}_{i j}^{C}+\hat{V}_{i j}^{B},
$$

where the Coulomb interaction operator is $\hat{V}_{i j}^{C}=1 / r_{i j}$, and $\omega_{i j}=\left(\varepsilon_{i}-\varepsilon_{j}\right) / c$ is the frequency of the one virtual photon exchanged $^{39}\left(\varepsilon_{i}\right.$ and $\varepsilon_{j}$ are the orbital energies of interacting electrons).

The unretarded (instantaneous) parts are obtained by applying the long wavelength approximation: $\omega_{i j} \rightarrow 0$. Then within the Feynman gauge, the Coulomb-Gaunt interaction terms are

\begin{tabular}{|c|c|c|c|c|c|c|c|c|c|}
\hline & \multicolumn{3}{|c|}{ Present } & \multicolumn{2}{|c|}{ Reference 16} & \multicolumn{2}{|c|}{ Reference 22} & \multirow{2}{*}{$\begin{array}{c}\text { Reference } 24 \\
\text { PT }\end{array}$} & \multirow{2}{*}{$\begin{array}{c}\text { Reference } 27 \\
\text { SC }\end{array}$} \\
\hline & PT & $\mathrm{PT}^{*}$ & $\mathrm{SC}$ & $\mathrm{PT}^{*}$ & $\mathrm{SC}$ & $\mathrm{PT}^{*}$ & $\mathrm{SC}$ & & \\
\hline $1 s$ & 309.497 & 315.893 & 298.329 & 315.11 & 298.24 & 312.191 & 298.661 & 308.147 & 298.329 \\
\hline $2 s$ & 42.129 & 41.823 & 33.456 & 41.72 & 33.47 & 41.981 & 33.486 & 42.13 & 33.456 \\
\hline $3 s$ & 9.729 & 9.653 & 6.191 & 9.636 & 6.193 & 9.926 & 6.198 & 9.729 & 6.191 \\
\hline $4 s$ & 2.519 & 2.5 & 1.269 & 2.496 & 1.270 & 2.761 & 1.272 & 2.52 & 1.269 \\
\hline $5 s$ & 0.498 & 0.494 & 0.191 & 0.493 & 0.191 & 0.568 & 0.192 & 0.498 & 0.191 \\
\hline $6 s$ & 0.042 & 0.042 & 0.012 & 0.042 & 0.012 & 0.038 & 0.012 & 0.042 & 0.012 \\
\hline $2 p_{1 / 2}$ & 65.502 & 65.663 & 56.253 & 65.47 & 56.25 & 66.549 & 56.259 & 65.499 & 56.253 \\
\hline $3 p_{1 / 2}$ & 14.47 & 14.375 & 10.703 & 14.338 & 10.702 & 15.397 & 10.704 & 14.47 & 10.703 \\
\hline $4 p_{1 / 2}$ & 3.529 & 3.5 & 2.269 & 3.491 & 2.269 & 4.153 & 2.269 & 3.528 & 2.269 \\
\hline $5 p_{1 / 2}$ & 0.6 & 0.595 & 0.33 & 0.593 & 0.330 & 0.778 & 0.33 & 0.6 & 0.33 \\
\hline $2 p_{3 / 2}$ & 41.149 & 44.024 & 35.507 & 43.92 & 35.51 & 45.457 & 35.505 & 41.146 & 35.507 \\
\hline $3 p_{3 / 2}$ & 9.156 & 9.756 & 6.386 & 9.736 & 6.387 & 11.147 & 6.386 & 9.155 & 6.386 \\
\hline $4 p_{3 / 2}$ & 2.185 & 2.327 & 1.186 & 2.322 & 1.186 & 3.203 & 1.186 & 2.184 & 1.186 \\
\hline $5 p_{3 / 2}$ & 0.351 & 0.374 & 0.131 & 0.373 & 0.131 & 0.628 & 0.131 & 0.351 & 0.131 \\
\hline $3 d_{3 / 2}$ & 8.32 & 8.322 & 4.648 & 8.305 & 4.648 & 9.369 & 4.648 & 8.32 & 4.648 \\
\hline $4 d_{3 / 2}$ & 1.749 & 1.747 & 0.625 & 1.743 & 0.625 & 2.405 & 0.625 & 1.75 & 0.625 \\
\hline $5 d_{3 / 2}$ & 0.166 & 0.165 & 0.007 & 0.165 & 0.007 & 0.273 & 0.007 & 0.166 & 0.007 \\
\hline $3 d_{5 / 2}$ & 6.501 & 6.56 & 2.99 & 6.547 & 2.988 & 7.619 & 2.989 & 6.501 & 2.99 \\
\hline $4 d_{5 / 2}$ & 1.341 & 1.352 & 0.26 & 1.350 & 0.260 & 1.972 & 0.26 & 1.342 & 0.26 \\
\hline $5 d_{5 / 2}$ & 0.119 & 0.12 & -0.03 & 0.120 & -0.030 & 0.219 & -0.03 & 0.118 & -0.03 \\
\hline $4 f_{5 / 2}$ & 0.762 & 0.747 & -0.158 & 0.760 & -0.158 & 1.218 & -0.158 & 0.762 & -0.158 \\
\hline $4 f_{7 / 2}$ & 0.598 & 0.588 & -0.312 & 0.597 & -0.313 & 1.032 & -0.313 & 0.597 & -0.312 \\
\hline
\end{tabular}

TABLE I. Energy contribution per subshell of Breit interaction for ${ }_{80} \mathrm{Hg}$ (eV units), calculated with perturbational (PT) and self-consistent (SC) approaches. PT means the frequency-dependent Breit operator and PT* means the frequency-independent Breit operator [Eq. (7)]. 


$$
\hat{V}_{i j(F)}\left(\omega_{i j}=0\right)=\frac{1}{r_{i j}}\left(1-\alpha_{i} \cdot \alpha_{j}\right)=\hat{V}_{i j}^{C}+\hat{V}_{i j}^{G},
$$

where $-\left(\boldsymbol{\alpha}_{i} \cdot \boldsymbol{\alpha}_{j}\right) / r_{i j}$ is called the Gaunt (magnetic) term. ${ }^{40}$ Within the Coulomb gauge, the Coulomb-Breit terms are given as

$\hat{V}_{i j(C)}\left(\omega_{i j}=0\right)=\frac{1}{r_{i j}}\left(1-\frac{1}{2} \alpha_{i} \cdot \alpha_{j}-\frac{\left(\alpha_{i} \cdot r_{i j}\right)\left(\alpha_{j} \cdot r_{i j}\right)}{2 r_{i j}^{3}}\right)$.

In the above equation, the Breit $\hat{V}_{i j}^{B}$ operator is written in the zero-frequency limit $\left(\omega_{i j} \rightarrow 0\right)$ as

$$
\begin{aligned}
\hat{V}_{i j}^{B} & =-\frac{\alpha_{i} \cdot \alpha_{j}}{2 r_{i j}}-\frac{\left(\boldsymbol{\alpha}_{i} \cdot r_{i j}\right)\left(\boldsymbol{\alpha}_{j} \cdot r_{i j}\right)}{2 r_{i j}^{3}} \\
& =\underbrace{\frac{\boldsymbol{\alpha}_{i} \cdot \boldsymbol{\alpha}_{j}}{r_{i j}}}_{V_{G}}+\underbrace{\left(\frac{\boldsymbol{\alpha}_{i} \cdot \boldsymbol{\alpha}_{j}}{2 r_{i j}}-\frac{\left(\boldsymbol{\alpha}_{i} \cdot r_{i j}\right)\left(\boldsymbol{\alpha}_{j} \cdot r_{i j}\right)}{2 r_{i j}^{3}}\right)}_{V_{r e t}},
\end{aligned}
$$

where $V_{\text {ret }}$ is called the gauge term or, commonly but somewhat misleadingly, retardation part. In this equation, the frequency-dependent term of order $\mathrm{O}\left(\omega^{2}\right)$ is omitted.

Gorceix et al. ${ }^{36}$ and Gorceix and Indelicato ${ }^{46}$ have found that the interaction potentials derived in the Coulomb and Feynman gauges, respectively, lead to significantly different numerical results in a MCDF calculation. Sucher ${ }^{47}$ found that, in contrast to the Coulomb gauge, the non-perturbative use of potential in the Feynman gauge leads to energy levels which are already incorrect in order $\alpha^{4} \mathrm{~m}$, even if the frequency dependence is included. Lindroth and Mårtensson-Pendrill ${ }^{48}$ have examined the gauge dependence further and found that the discrepancy remains, even if one goes beyond the novirtual-pair approximation. This fact provides a quantitative argument for preferring the Coulomb gauge over the Feynman gauge. Lindgren ${ }^{49}$ demonstrated that within the no-virtual-pair approximation both the gauges yield the same energy shift to order $\mathrm{O}\left(\alpha^{2}\right.$ hartree) only if the irreducible part of the twophoton interaction in the Feynman gauge is also taken into account.

The zero-frequency approximation to the full transverse Breit interaction, i.e., Eq. (7), is more common in computations of many-electron atomic systems than the explicit frequencydependent form because of remedying the lack of covariance of the Dirac-Coulomb-Breit Hamiltonian. The differences of state energy calculated by using frequency-independent and frequency-dependent Breit operators are found to be small. ${ }^{16,36,50}$ For the super-heavy Rn-like ions with $Z=170$, Indelicato $^{51}$ found that the frequency-dependent term contribute $-1.3 \%$ (having opposite sign) of the total Breit correction to $1 s$ binding energy. The finite $\omega$ corrections were studied in the work of Beatham et al. ${ }^{52}$ in the case of $K \alpha_{1,2}$

\begin{tabular}{|c|c|c|c|c|c|c|c|c|c|c|c|c|}
\hline & \multicolumn{3}{|c|}{$\mathrm{Zn}$} & \multicolumn{4}{|c|}{$\mathrm{Xe}$} & \multicolumn{5}{|c|}{ No } \\
\hline & PT & $\mathrm{PT}^{*}$ & $\mathrm{SC}$ & PT & $\mathrm{PT}^{*}$ & $\mathrm{SC}$ & $\begin{array}{c}\text { Reference } 12 \\
\text { SC }\end{array}$ & PT & $\mathrm{PT}^{*}$ & SC & $\begin{array}{c}\text { Reference } 27 \\
\text { SC }\end{array}$ & $\begin{array}{c}\text { Reference } 28 \\
\text { SC }\end{array}$ \\
\hline $1 s$ & 13.135 & 13.171 & 11.875 & 86.036 & 86.883 & 80.712 & 80.709 & 718.755 & 739.879 & 703.058 & 703.145 & 703.142 \\
\hline $2 s$ & 1.324 & 1.322 & 0.851 & 10.432 & 10.392 & 7.634 & 7.633 & 107.558 & 106.498 & 88.649 & 88.669 & 88.682 \\
\hline $3 s$ & 0.195 & 0.195 & 0.090 & 2.110 & 2.101 & 1.101 & 1.101 & 26.547 & 26.297 & 18.434 & 18.437 & 18.436 \\
\hline $4 s$ & 0.008 & 0.008 & 0.003 & 0.447 & 0.445 & 0.153 & 0.153 & 7.795 & 7.720 & 4.561 & 4.562 & 4.562 \\
\hline $5 s$ & & & & 0.019 & 0.058 & 0.008 & 0.007 & 2.270 & 2.248 & 1.140 & 1.140 & 1.140 \\
\hline $6 s$ & & & & & & & & 0.700 & 0.693 & 0.213 & 0.213 & 0.213 \\
\hline $7 s$ & & & & & & & & 0.255 & 0.252 & 0.007 & 0.007 & 0.007 \\
\hline $2 p_{1 / 2}$ & 2.018 & 2.020 & 1.503 & 16.066 & 16.106 & 13.045 & 13.045 & 169.722 & 169.395 & 149.061 & 149.064 & 149.064 \\
\hline $3 p_{1 / 2}$ & 0.257 & 0.255 & 0.160 & 3.072 & 3.069 & 2.034 & 2.034 & 39.810 & 39.138 & 30.699 & 30.700 & 30.700 \\
\hline $4 p_{1 / 2}$ & & & & 0.591 & 0.590 & 0.314 & 0.315 & 11.133 & 10.919 & 7.654 & 7.655 & 7.655 \\
\hline $5 p_{1 / 2}$ & & & & 0.014 & 0.057 & 0.020 & 0.020 & 3.047 & 2.986 & 1.852 & 1.852 & 1.853 \\
\hline $6 p_{1 / 2}$ & & & & & & & & 0.897 & 0.879 & 0.295 & 0.295 & 0.296 \\
\hline $2 p_{3 / 2}$ & 1.486 & 1.502 & 0.976 & 11.407 & 11.781 & 8.815 & 8.814 & 88.814 & 98.656 & 81.813 & 81.812 & 81.814 \\
\hline $3 p_{3 / 2}$ & 0.187 & 0.188 & 0.085 & 2.168 & 2.236 & 1.228 & 1.228 & 21.339 & 23.544 & 16.336 & 16.336 & 16.335 \\
\hline $4 p_{3 / 2}$ & & & & 0.413 & 0.426 & 0.151 & 0.151 & 5.832 & 6.437 & 3.629 & 3.629 & 3.629 \\
\hline $5 p_{3 / 2}$ & & & & 0.076 & 0.039 & 0.003 & 0.003 & 1.573 & 1.737 & 0.764 & 0.764 & 0.764 \\
\hline $6 p_{3 / 2}$ & & & & & & & & 0.460 & 0.508 & 0.083 & 0.083 & 0.083 \\
\hline $3 d_{3 / 2}$ & 0.086 & 0.084 & 0.005 & 1.704 & 1.704 & 0.630 & 0.630 & 20.982 & 21.036 & 13.268 & 13.268 & 13.268 \\
\hline $4 d_{3 / 2}$ & & & & 0.245 & 0.243 & 0.006 & 0.006 & 5.306 & 5.306 & 2.459 & 2.459 & 2.459 \\
\hline $5 d_{3 / 2}$ & & & & & & & & 1.260 & 1.259 & 0.378 & 0.378 & 0.378 \\
\hline $3 d_{5 / 2}$ & 0.064 & 0.062 & -0.022 & 1.337 & 1.342 & 0.277 & 0.277 & 16.115 & 16.365 & 8.979 & 8.979 & 8.980 \\
\hline $4 d_{5 / 2}$ & & & & 0.187 & 0.187 & -0.052 & -0.052 & 4.025 & 4.080 & 1.381 & 1.381 & 1.381 \\
\hline $5 d_{5 / 2}$ & & & & & & & & 0.946 & 0.959 & 0.133 & 0.133 & 0.134 \\
\hline $4 f_{5 / 2}$ & & & & & & & & 2.866 & 2.862 & 0.293 & 0.293 & 0.293 \\
\hline $5 f_{5 / 2}$ & & & & & & & & 0.001 & 0.001 & -0.088 & -0.088 & -0.088 \\
\hline $4 f_{7 / 2}$ & & & & & & & & 2.284 & 2.290 & -0.217 & -0.218 & -0.217 \\
\hline $5 f_{7 / 2}$ & & & & & & & & 0.002 & 0.002 & -0.156 & -0.156 & -0.156 \\
\hline
\end{tabular}

TABLE II. Energy contribution per subshell of Breit interaction for ${ }_{30} \mathrm{Zn},{ }_{54} \mathrm{Xe}$, and ${ }_{102}$ No, calculated with perturbational (PT) and self-consistent (SC) approaches. PT means the frequency-dependent Breit operator and PT* means the frequency-independent Breit operator [Eq. (7)]. 
TABLE III. $1 s$ ionization potential for $\mathrm{Xe}(\mathrm{eV})$.

\begin{tabular}{|c|c|c|c|}
\hline & Xe (neutral) & $\mathrm{Xe}(1 \mathrm{~s}$ hole $)$ & IP (1s) \\
\hline DC & -202639.438 & -167952.784 & 34686.654 \\
\hline+ Breit (SC) & 155.104 & 73.597 & -81.506 \\
\hline mag. & 174.930 & 86.169 & -88.761 \\
\hline ret. & -18.073 & -11.595 & 6.478 \\
\hline$\omega$-dep. & -1.753 & -0.976 & 0.776 \\
\hline$+\operatorname{Breit}(\mathrm{PT})$ & 155.395 & 73.687 & -81.708 \\
\hline mag. & 175.261 & 86.278 & -88.983 \\
\hline ret. & -18.107 & -11.613 & 6.494 \\
\hline$\omega$-dep. & -1.759 & -0.978 & 0.781 \\
\hline$+\mathrm{SE}$ & 118.650 & 70.558 & -48.092 \\
\hline$+\mathrm{VP}$ & -16.520 & -9.575 & 6.946 \\
\hline Total (SC) & -202382.205 & -167818.203 & 34564.001 \\
\hline Total (PT) & -202381.636 & -167818.065 & -34563.571 \\
\hline \multicolumn{4}{|l|}{ Theory } \\
\hline \multicolumn{3}{|c|}{ Indelicato et al. ${ }^{41}$} & 34566.52 \\
\hline \multicolumn{3}{|c|}{ Deslattes et $a l^{42}$} & $34566.5(26)$ \\
\hline \multicolumn{3}{|c|}{ Niskanen et $_{\text {al. }}{ }^{26}$} & 34598.0 \\
\hline \multicolumn{3}{|c|}{ Barysz and Syrocki ${ }^{43}$} & 34532.59 \\
\hline \multicolumn{4}{|l|}{ Experiment } \\
\hline \multicolumn{3}{|c|}{ Bearden and Burr ${ }^{44}$} & $34561.4(11)$ \\
\hline \multicolumn{3}{|c|}{ Deutsch et al. ${ }^{45}$} & 34563.4 \\
\hline \multicolumn{3}{|c|}{ Deslattes et al. $^{42}$} & $34565.13(33)$ \\
\hline
\end{tabular}

and $K^{h} \alpha_{1,2}$ lines of $\mathrm{Hg}$. They found that the finite $\omega$ corrections contribute $-2.4 \%$ and $-1.8 \%$ to the total Breit energy contribution in the case of single $1 s$ - and $2 p$-shell ionized $\mathrm{Hg}$ atoms, respectively.

The Breit interaction can be included in two general ways: in the self-consistent field process, such as in the MCDFGME code, ${ }^{36,53-55}$ or in the perturbational approach, such as in the GRASP code. ${ }^{56,57}$

TABLE IV. $1 s$ ionization potential for Rn $(\mathrm{eV})$.

\begin{tabular}{lrrr}
\hline \hline & Rn (neutral) & Rn (1s hole) & \multicolumn{1}{c}{ IP (1s) } \\
\hline DC & -642240.927 & -543260.674 & 98980.252 \\
+Breit (SC) & 778.104 & 399.690 & -378.414 \\
mag. & 891.970 & 473.801 & -418.169 \\
ret. & -94.475 & -63.376 & 31.099 \\
$\omega$-dep. & -19.391 & -10.734 & 8.657 \\
+Breit (PT) & 780.425 & 400.482 & -379.943 \\
mag. & 894.673 & 474.767 & -419.906 \\
ret. & -94.747 & -63.525 & 31.222 \\
$\omega$-dep. & -19.501 & -10.760 & 8.741 \\
+SE & 753.827 & 482.054 & -271.773 \\
+ VP & -156.097 & -94.204 & 61.894 \\
Total (SC) & -640931.677 & -542527.246 & 98404.431 \\
Total (PT) & -640928.327 & -542526.438 & 98401.889 \\
\hline
\end{tabular}

\section{Theory}

Indelicato et al. ${ }^{41}$

Deslattes et al. $^{42}$

Niskanen et al. $^{26}$

Experiment

Bearden and Burr ${ }^{44}$

Deslattes et ${ }^{42}$
An atomic state function (ASF) with the total angular momentum $J$, its $z$-projection $M$, and parity $p$ is assumed in the form

$$
\Psi_{s}\left(J M^{p}\right)=\sum_{m} c_{m}(s) \Phi\left(\gamma_{m} J M^{p}\right),
$$

where $\Phi\left(\gamma_{m} J M^{p}\right)$ are configuration state functions (CSFs), $c_{m}(s)$ are the configuration mixing coefficients for state $s, \gamma_{m}$ represents all information required to uniquely define a certain CSF.

The CSF is a Slater determinant of Dirac 4-component bispinors,

$$
\Phi\left(\gamma_{m} J M^{p}\right)=\sum_{i} d_{i}\left|\begin{array}{ccc}
\psi_{1}(1) & \cdots & \psi_{1}(N) \\
\vdots & \ddots & \vdots \\
\psi_{N}(1) & \cdots & \psi_{N}(N)
\end{array}\right|
$$

where $\psi_{i}$ is the one-electron wavefunction and $d_{i}$ coefficients are determined by considering that the CSF is an eigenstate of $\hat{J}^{2}$ and $\hat{J}_{z}$. The one-electron wavefunction is defined as

$$
\psi=\frac{1}{r}\left(\begin{array}{c}
P_{n, \kappa}(r) \cdot \Omega_{\kappa, j}^{m_{j}}(\theta, \phi) \\
i Q_{n, K}(r) \cdot \Omega_{-\kappa, j}^{m_{j}}(\theta, \phi)
\end{array}\right),
$$

\begin{tabular}{|c|c|c|c|c|c|c|c|}
\hline$Z$ & $1 s_{1 / 2}$ & $2 s_{1 / 2}$ & $2 p_{1 / 2}$ & $2 p_{3 / 2}$ & $3 s_{1 / 2}$ & $3 p_{1 / 2}$ & $3 p_{3 / 2}$ \\
\hline 30 & 0.4364 & 0.0313 & 0.0552 & 0.0359 & 0.0033 & 0.0059 & 0.0031 \\
\hline 32 & 0.5380 & 0.0394 & 0.0698 & 0.0456 & 0.0039 & 0.0074 & 0.0039 \\
\hline 36 & 0.7893 & 0.0609 & 0.1074 & 0.0711 & 0.0062 & 0.0123 & 0.0066 \\
\hline 48 & 2.0170 & 0.1810 & 0.3117 & 0.2109 & 0.0246 & 0.0458 & 0.0274 \\
\hline 50 & 2.3046 & 0.2105 & 0.3616 & 0.2447 & 0.0291 & 0.0541 & 0.0324 \\
\hline 54 & 2.9661 & 0.2805 & 0.4794 & 0.3239 & 0.0404 & 0.0747 & 0.0451 \\
\hline 62 & 4.6759 & 0.4734 & 0.8001 & 0.5359 & 0.0783 & 0.1386 & 0.0855 \\
\hline 72 & 7.6824 & 0.8271 & 1.3913 & 0.9067 & 0.1482 & 0.2572 & 0.1577 \\
\hline 80 & 10.9634 & 1.2295 & 2.0673 & 1.3049 & 0.2275 & 0.3933 & 0.2347 \\
\hline 82 & 11.9291 & 1.3508 & 2.2713 & 1.4201 & 0.2517 & 0.4347 & 0.2571 \\
\hline 86 & 14.0590 & 1.6247 & 2.7312 & 1.6733 & 0.3084 & 0.5299 & 0.3082 \\
\hline 104 & 27.7673 & 3.5408 & 5.9576 & 3.2104 & 0.7421 & 1.2328 & 0.6446 \\
\hline 112 & 36.8476 & 4.9417 & 8.3446 & 4.1270 & 1.0724 & 1.7617 & 0.8494 \\
\hline 114 & 39.5151 & 5.3734 & 9.0865 & 4.3804 & 1.1751 & 1.9265 & 0.9059 \\
\hline 118 & 45.4271 & 6.3676 & 10.8087 & 4.9232 & 1.4167 & 2.3132 & 1.0301 \\
\hline$Z$ & $3 d_{3 / 2}$ & $3 d_{5 / 2}$ & $4 s_{1 / 2}$ & $4 p_{1 / 2}$ & $4 p_{3 / 2}$ & $4 d_{3 / 2}$ & $4 d_{5 / 2}$ \\
\hline 30 & 0.0002 & -0.0008 & 0.0001 & & & & \\
\hline 32 & 0.0001 & -0.0012 & 0.0001 & 0.0001 & 0.0003 & & \\
\hline 36 & 0.0008 & -0.0016 & 0.0003 & 0.0006 & 0.0001 & & \\
\hline 48 & 0.0123 & 0.0042 & 0.0035 & 0.0064 & 0.0031 & 0.0001 & -0.0009 \\
\hline 50 & 0.0152 & 0.0057 & 0.0039 & 0.0077 & 0.0036 & -0.0001 & -0.0014 \\
\hline 54 & 0.0232 & 0.0102 & 0.0056 & 0.0116 & 0.0055 & 0.0002 & -0.0019 \\
\hline 62 & 0.0535 & 0.0296 & 0.0151 & 0.0277 & 0.0143 & 0.0078 & -0.0002 \\
\hline 72 & 0.1091 & 0.0684 & 0.0302 & 0.0528 & 0.0289 & 0.0142 & 0.0057 \\
\hline 80 & 0.1708 & 0.1099 & 0.0467 & 0.0834 & 0.0436 & 0.0230 & 0.0096 \\
\hline 82 & 0.1891 & 0.1220 & 0.0515 & 0.0927 & 0.0476 & 0.0253 & 0.0104 \\
\hline 86 & 0.2314 & 0.1509 & 0.0646 & 0.1159 & 0.0584 & 0.0324 & 0.0139 \\
\hline 104 & 0.5273 & 0.3570 & 0.1849 & 0.3096 & 0.1435 & 0.0982 & 0.0551 \\
\hline 112 & 0.7133 & 0.4841 & 0.2784 & 0.4583 & 0.1938 & 0.1386 & 0.0791 \\
\hline 114 & 0.7649 & 0.5187 & 0.3071 & 0.5045 & 0.2069 & 0.1491 & 0.0848 \\
\hline 118 & 0.8791 & 0.5958 & 0.3779 & 0.6161 & 0.2384 & 0.1751 & 0.1005 \\
\hline
\end{tabular}

TABLE V. SC Breit interaction contribution to the orbital energy for selected atoms (hartree unit). 
where $\Omega_{\kappa, j}^{m_{j}}(\theta, \phi)$ is a angular 2-component spinor and $P_{n, \kappa}(r)$ and $Q_{n, \kappa}(r)$ are the large and small radial parts of the wavefunction, respectively.

\section{B. Computational details}

Present results for Breit interaction contribution were obtained by performing calculations with the $\mathrm{MCDFGME}^{53-55}$ code for the self-consistent approach (using the retarded Breit operator) and by the GRASPK code ${ }^{57,58}$ for the perturbational approach (using both retarded and unretarded Breit operators).

The Breit interaction contribution to the orbital energy of the $n \kappa$ subshell has been obtained by the subtraction of the orbital energy computed by means of self-consistent DiracFock equations using the Coulomb operator [only the first part in Eq. (4)], $\varepsilon^{D C}$, from the orbital energy computed by means of self-consistent Dirac-Fock equations using the Coulomb-Breit operator [all parts of Eq. (4)], $\varepsilon^{D C B}$, i.e.,

$$
\varepsilon_{n \kappa}^{B r e i t}=\varepsilon_{n \kappa}^{D C B}-\varepsilon_{n \kappa}^{D C} .
$$

\section{RESULTS}

We first shall compare the Breit contributions to the orbital energy in a many-electron atomic system calculated by a perturbation theory approach and by a self-consistency procedure. Although similar comparisons were presented before in the literature, ${ }^{16,22}$ we use experimental data to examine which approach is more accurate. Then we will examine the $Z$ dependence of Breit contribution to orbital energies. Although
TABLE VI. Fitting coefficients for $Z$-dependence of the Breit interaction (see text for details).

\begin{tabular}{lccc}
\hline \hline Subshell & $a$ & $b$ & $R^{2}$ \\
\hline $1 s$ & $1.370 \times 10^{-6}$ & 3.627 & 0.99961 \\
$2 s$ & $1.039 \times 10^{-8}$ & 4.237 & 0.99926 \\
$3 s$ & $1.908 \times 10^{-10}$ & 4.761 & 0.99942 \\
$4 s$ & $1.709 \times 10^{-12}$ & 5.473 & 0.99963 \\
$2 p_{1 / 2}$ & $1.557 \times 10^{-8}$ & 4.263 & 0.99908 \\
$3 p_{1 / 2}$ & $6.649 \times 10^{-9}$ & 4.601 & 0.99937 \\
$4 p_{1 / 2}$ & $9.472 \times 10^{-12}$ & 5.216 & 0.99963 \\
$2 p_{3 / 2}$ & $3.836 \times 10^{-7}$ & 3.431 & 0.99997 \\
$3 p_{3 / 2}$ & $1.201 \times 10^{-8}$ & 3.830 & 0.99988 \\
$4 p_{3 / 2}$ & $2.109 \times 10^{-10}$ & 4.372 & 0.99945 \\
\hline \hline
\end{tabular}

the $Z$-dependence of Breit contribution has been studied in the case of total atomic energy ${ }^{24}$ and in the cases of single and double $1 s$ electron ionization energies; ${ }^{26}$ according to our knowledge, no-one focused on orbital energies. Next, we will study relations between magnetic and retardation parts of the Breit interaction. Finally, we will show results from the DiRAC code $^{59}$ for atomic and molecular systems.

\section{A. Breit contributions from perturbational and self-consistent procedures}

The comparison between perturbational (PT) and selfconsistent field (SC) computational approaches of the Breit interaction energy contribution per subshell is presented in Tables I and II. The PT numbers are presented in the case

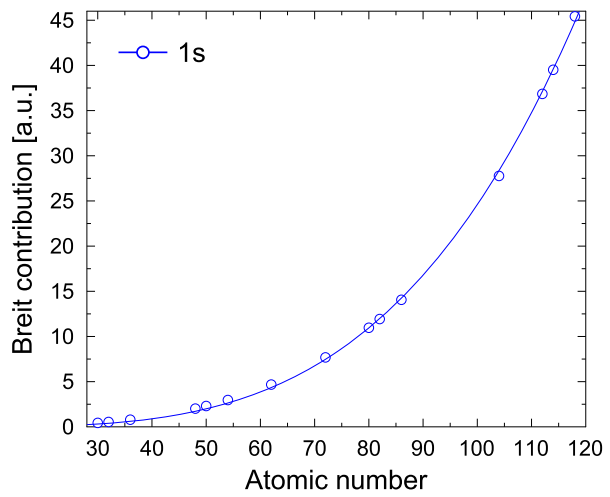

(a)

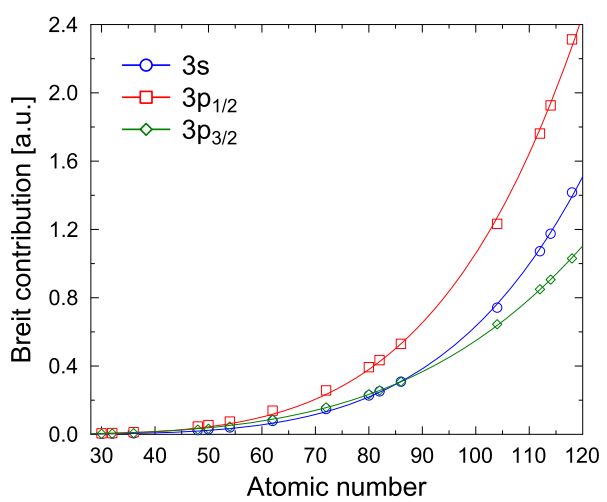

(c)

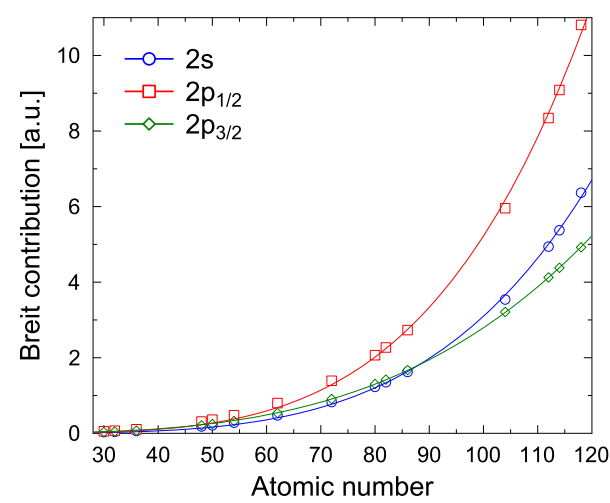

(b)

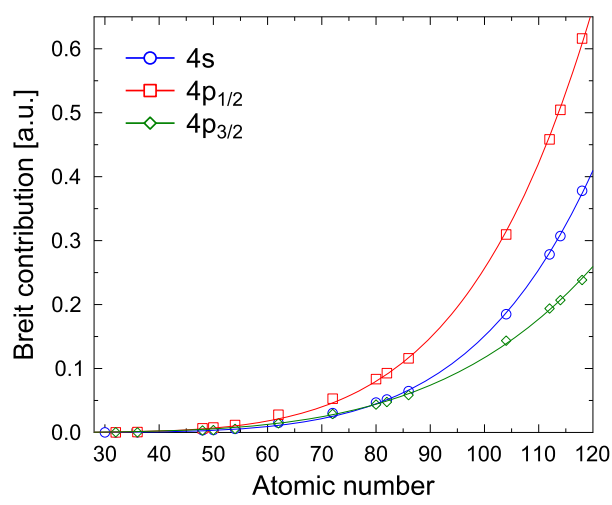

(d)
FIG. 1. Breit contributions to DiracCoulomb orbital energy for $n l$ subshells (points) and fit lines to them. 
of frequency-dependent Breit operator, "PT," and in the case of frequency-independent Breit operator, "PT*." Our results of the Breit contributions to the orbital energies for the $\mathrm{Hg}$ atom are similar to the studies by Lindroth et al. ${ }^{16}$ by Thierfelder and Schwerdtfeger, ${ }^{22}$ by Mann and Johnson, ${ }^{24}$ and by Parpia et al. ${ }^{27}$ in each, SC, PT, and $\mathrm{PT}^{*}$ case. We also performed similar calculations for $\mathrm{Zn}, \mathrm{Xe}$, and $\mathrm{No}$, in order to have more comprehensive insights into PT vs SC treatment of the Breit contributions. As one can see from Tables I and II, our results are very close to the data presented in the literature, ${ }^{12,16,22,24,27,28}$ for both PT and SC approaches. The PT contribution is higher than the SC one in all studied cases. The PT-SC difference decreases when $Z$ increases (from $10 \%$ for $1 s$ of $\mathrm{Zn}$ to $5 \%$ for $1 s$ of No) and increases rapidly when the shell number $n$ of orbital increases (no case: $5 \%$ for $1 s$, $20 \%$ for $2 s$, and $43 \%$ for $3 s$ ). However, if changes of atomic (or molecular) orbitals due to the Breit term (see, e.g., Ref. 60) are considered, a variational self-consistent treatment is required. As one can see, the finite $\omega$ corrections contribute to the orbital Breit energy contribution more in the case of $p_{3 / 2}$ orbital than in the case of $s$ or $p_{1 / 2}$ orbitals, but all do not exceed 10\%-compared to columns "PT" and "PT"" in Tables I and II.

In order to keep track on how accurate our results are as compared with experiments, we performed the calculation of $1 s$ ionization potential of $\mathrm{Xe}$ and $\mathrm{Rn}$, which were recently studied by Niskanen et al. ${ }^{26}$ and by Barysz and Syrocki; ${ }^{43}-$ see Tables III and IV. The "DC" (Dirac-Coulomb energy only), "Breit (SC)," "Breit (PT)," "SE" (in the Welton model), and "VP" (Uehling potential) are contributions calculated by the MCDFGME code. Total values also include higher order SE and VP terms by means of the McDFGME code. ${ }^{2}$ The "total (SC)" means "DC" + "Breit (SC)" + "SE" + "VP" + higher order contributions. The "total (PT)" is similar to the "total (SC)," but the "Breit (SC)" contribution is replaced by the "Breit (PT)" contribution. The Breit correction is divided into three contributions: "mag."-magnetic (Gaunt) part, "ret."frequency independent retardation part [ $V_{r e t}$ in Eqs. (7)], and " $\omega$-dep."-frequency dependent retardation part. As one can see from Table III, our "total (SC)" results are even closer to the experiment than very accurate result of Indelicato et al. ${ }^{41}$ and Deslattes et al. ${ }^{42}$ (both obtained using the earlier version of the MCDFGME code and SC treatment of the Breit term). The "total (PT)" values are only a little farther away from the experiment but still in very good agreement. Then we decided to use SC Breit values in our study. One can see that the difference between SC and PT results is bigger in the case of orbital energies (see Tables I and II) than in the case of total state energies.

\section{B. Z-dependence of Breit contribution}

In Table $\mathrm{V}$, the contribution of the Breit interaction to the orbital energy for selected atoms with $30 \leq \mathrm{Z} \leq 118$ has been collected. Their $Z$-dependencies have been examined by fitting Breit contributions to the orbital $n s(n=1-4)$ and $n^{\prime} p_{1 / 2}$ and $n^{\prime} p_{3 / 2}\left(n^{\prime}=2-4\right)$ energies, with the function

$$
\varepsilon_{n l}^{\text {Breit }}(Z)=a \times Z^{b} .
$$

TABLE VII. Breit energy contributions to the Dirac-Coulomb orbital energy ratio (all numbers are multiplied by $10^{3}$, e.g., -1.2199 means $-1.2199 \times 10^{-3}$ ).

\begin{tabular}{lccccccc}
\hline \hline$Z$ & $1 s_{1 / 2}$ & $2 s_{1 / 2}$ & $2 p_{1 / 2}$ & $2 p_{3 / 2}$ & $3 s_{1 / 2}$ & $3 p_{1 / 2}$ & $3 p_{3 / 2}$ \\
\hline 30 & -1.2199 & -0.6901 & -1.3901 & -0.9229 & -0.5691 & -1.4896 & -0.8170 \\
32 & -1.3088 & -0.7372 & -1.4749 & -0.9879 & -0.5299 & -1.3990 & -0.7568 \\
36 & -1.4901 & -0.8450 & -1.6562 & -1.1309 & -0.5513 & -1.4220 & -0.7896 \\
48 & -2.0428 & -1.2085 & -2.2417 & -1.5989 & -0.8404 & -1.8356 & -1.1599 \\
50 & -2.1353 & -1.2657 & -2.3351 & -1.6720 & -0.8664 & -1.8754 & -1.1900 \\
54 & -2.3223 & -1.3856 & -2.5275 & -1.8229 & -0.9404 & -1.9844 & -1.2772 \\
62 & -2.7010 & -1.6492 & -2.9480 & -2.1507 & -1.2110 & -2.3936 & -1.6051 \\
72 & -3.1793 & -1.9782 & -3.4920 & -2.5576 & -1.5208 & -2.8988 & -1.9923 \\
80 & -3.5662 & -2.2344 & -3.9238 & -2.8668 & -1.7092 & -3.2072 & -2.2026 \\
82 & -3.6639 & -2.2978 & -4.0311 & -2.9411 & -1.7512 & -3.2739 & -2.2442 \\
86 & -3.8611 & -2.4293 & -4.2520 & -3.0924 & -1.8486 & -3.4214 & -2.3398 \\
104 & -4.7804 & -3.0857 & -5.3620 & -3.7923 & -2.4394 & -4.2899 & -2.8778 \\
112 & -5.2121 & -3.4207 & -5.9364 & -4.0979 & -2.7444 & -4.7360 & -3.0890 \\
114 & -5.3235 & -3.5113 & -6.0922 & -4.1724 & -2.8257 & -4.8555 & -3.1350 \\
118 & -5.5518 & -3.7062 & -6.4278 & -4.3241 & -3.0077 & -5.1213 & -3.2359 \\
\hline$Z$ & $3 d_{3 / 2}$ & $3 d_{5 / 2}$ & $4 s_{1 / 2}$ & $4 p_{1 / 2}$ & $4 p_{3 / 2}$ & $4 d_{3 / 2}$ & $4 d_{5 / 2}$ \\
\hline 30 & -0.2611 & 1.0941 & -0.4185 & & & & \\
32 & -0.0931 & 0.7722 & -0.2056 & -0.3897 & -0.8719 & & \\
36 & -0.2054 & 0.4273 & -0.2112 & -1.0382 & -0.1307 & & \\
48 & -0.7692 & -0.2648 & -0.7334 & -1.9520 & -1.0293 & -0.1144 & 1.2454 \\
50 & -0.7986 & -0.3015 & -0.6649 & -1.8076 & -0.9214 & 0.0574 & 1.0821 \\
54 & -0.8897 & -0.3983 & -0.6675 & -1.7911 & -0.9272 & -0.0847 & 0.7250 \\
62 & -1.2821 & -0.7275 & -1.1044 & -2.5216 & -1.4420 & -1.3927 & 0.0305 \\
72 & -1.6867 & -1.0922 & -1.4554 & -3.0769 & -1.9363 & -1.5775 & -0.6668 \\
80 & -1.9100 & -1.2773 & -1.5222 & -3.1913 & -1.9645 & -1.5526 & -0.6803 \\
82 & -1.9537 & -1.3133 & -1.5177 & -3.1773 & -1.9320 & -1.5007 & -0.6462 \\
86 & -2.0557 & -1.4004 & -1.5634 & -3.2186 & -1.9383 & -1.5045 & -0.6798 \\
104 & -2.6611 & -1.9148 & -2.1807 & -4.0296 & -2.4267 & -2.1017 & -1.2615 \\
112 & -2.9010 & -2.1103 & -2.4541 & -4.3971 & -2.5376 & -2.2324 & -1.3741 \\
114 & -2.9533 & -2.1512 & -2.5182 & -4.4847 & -2.5440 & -2.2401 & -1.3770 \\
118 & -3.0668 & -2.2427 & -2.6814 & -4.7027 & -2.5905 & -2.2962 & -1.4291 \\
\hline \hline & & & & & & & \\
\hline
\end{tabular}

Coefficients $a$ and $b$ have been collected in Table VI (also $R^{2}$ fitting parameters have been presented). The fitting has been performed by means of the Nelder-Mead simplex algorithm implemented in the SciDAVis program. ${ }^{61}$

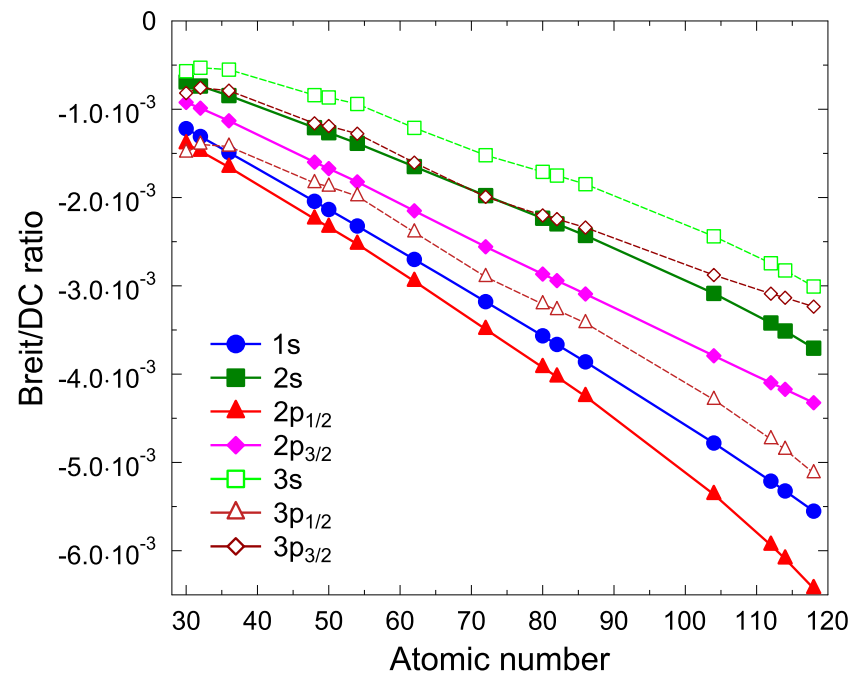

FIG. 2. Breit energy contributions to the Dirac-Coulomb orbital energy ratio for selected subshells. 
TABLE VIII. Magnetic (Gaunt) and retardation parts of Breit contributions to the Dirac-Coulomb orbital energy for selected atoms (hartree unit).

\begin{tabular}{|c|c|c|c|c|c|c|c|c|}
\hline$Z$ & & $1 s_{1 / 2}$ & $2 s_{1 / 2}$ & $2 p_{1 / 2}$ & $2 p_{3 / 2}$ & $3 s_{1 / 2}$ & $3 p_{1 / 2}$ & $3 p_{3 / 2}$ \\
\hline \multirow[t]{2}{*}{10} & mag. & $1.13 \times 10^{-2}$ & $1.79 \times 10^{-4}$ & $4.40 \times 10^{-4}$ & $7.11 \times 10^{-5}$ & & & \\
\hline & ret. & $-5.40 \times 10^{-5}$ & $4.13 \times 10^{-5}$ & $-3.23 \times 10^{-5}$ & $-3.22 \times 10^{-5}$ & & & \\
\hline \multirow[t]{2}{*}{18} & mag. & $8.44 \times 10^{-2}$ & $3.73 \times 10^{-3}$ & $7.82 \times 10^{-3}$ & $4.60 \times 10^{-3}$ & $1.30 \times 10^{-4}$ & $3.54 \times 10^{-4}$ & $5.81 \times 10^{-5}$ \\
\hline & ret. & $-3.32 \times 10^{-3}$ & $1.07 \times 10^{-4}$ & $-7.56 \times 10^{-4}$ & $-7.55 \times 10^{-4}$ & $3.09 \times 10^{-5}$ & $-3.14 \times 10^{-5}$ & $-3.14 \times 10^{-5}$ \\
\hline \multirow[t]{2}{*}{36} & mag. & 0.8417 & 0.0634 & 0.1204 & 0.0841 & $6.24 \times 10^{-3}$ & $1.38 \times 10^{-2}$ & $8.09 \times 10^{-3}$ \\
\hline & ret. & -0.0524 & -0.0025 & -0.0130 & -0.0129 & $-5.10 \times 10^{-5}$ & $-1.54 \times 10^{-3}$ & $-1.53 \times 10^{-3}$ \\
\hline \multirow[t]{2}{*}{54} & mag. & 3.1910 & 0.3006 & 0.5393 & 0.3836 & $4.25 \times 10^{-2}$ & $8.38 \times 10^{-2}$ & $5.41 \times 10^{-2}$ \\
\hline & ret. & -0.2249 & -0.0201 & -0.0599 & -0.0597 & $-2.06 \times 10^{-3}$ & $-9.03 \times 10^{-3}$ & $-9.01 \times 10^{-3}$ \\
\hline \multirow[t]{2}{*}{86} & mag. & 15.1617 & 1.7830 & 3.0421 & 1.9849 & 0.3393 & 0.5894 & 0.3678 \\
\hline & ret. & -1.1027 & -0.1583 & -0.3109 & -0.3116 & -0.0309 & -0.0594 & -0.0596 \\
\hline \multirow[t]{2}{*}{118} & mag. & 48.5109 & 7.1312 & 11.6743 & 5.8557 & 1.6143 & 2.5255 & 1.2324 \\
\hline & ret. & -3.0836 & -0.7636 & -0.8656 & -0.9325 & -0.1976 & -0.2123 & -0.2023 \\
\hline$Z$ & & $3 d_{3 / 2}$ & $3 d_{5 / 2}$ & $4 s_{1 / 2}$ & $4 p_{1 / 2}$ & $4 p_{3 / 2}$ & $4 d_{3 / 2}$ & $4 d_{5 / 2}$ \\
\hline \multirow[t]{2}{*}{36} & mag. & $5.79 \times 10^{-4}$ & $-1.79 \times 10^{-3}$ & $1.96 \times 10^{-4}$ & $6.17 \times 10^{-4}$ & $1.22 \times 10^{-4}$ & & \\
\hline & ret. & $1.97 \times 10^{-4}$ & $1.97 \times 10^{-4}$ & $5.45 \times 10^{-5}$ & $-5.49 \times 10^{-5}$ & $-5.52 \times 10^{-5}$ & & \\
\hline \multirow[t]{2}{*}{54} & mag. & $2.47 \times 10^{-2}$ & $1.16 \times 10^{-2}$ & $5.79 \times 10^{-3}$ & $1.30 \times 10^{-2}$ & $6.99 \times 10^{-3}$ & $5.90 \times 10^{-5}$ & $-2.08 \times 10^{-3}$ \\
\hline & ret. & $-1.52 \times 10^{-3}$ & $-1.48 \times 10^{-3}$ & $-1.65 \times 10^{-4}$ & $-1.45 \times 10^{-3}$ & $-1.44 \times 10^{-3}$ & $1.71 \times 10^{-4}$ & $1.69 \times 10^{-4}$ \\
\hline \multirow[t]{2}{*}{86} & mag. & 0.2581 & 0.1767 & 0.0715 & 0.1295 & 0.0713 & 0.0360 & 0.0173 \\
\hline & ret. & -0.0267 & -0.0258 & -0.0069 & -0.0135 & -0.0129 & -0.0036 & -0.0034 \\
\hline \multirow[t]{2}{*}{118} & mag. & 0.9922 & 0.7029 & 0.4332 & 0.6752 & 0.2892 & 0.1959 & 0.1204 \\
\hline & ret. & -0.1131 & -0.1070 & -0.0553 & -0.0591 & -0.0508 & -0.0207 & -0.0199 \\
\hline$Z$ & & $4 f_{5 / 2}$ & $4 f_{7 / 2}$ & $5 s_{1 / 2}$ & $5 p_{1 / 2}$ & $5 p_{3 / 2}$ & $5 d_{3 / 2}$ & $5 d_{5 / 2}$ \\
\hline \multirow[t]{2}{*}{54} & mag. & & & $2.29 \times 10^{-4}$ & $8.22 \times 10^{-4}$ & $1.63 \times 10^{-4}$ & & \\
\hline & ret. & & & $6.05 \times 10^{-5}$ & $-6.91 \times 10^{-5}$ & $-6.99 \times 10^{-5}$ & & \\
\hline \multirow[t]{2}{*}{86} & mag. & $-9.87 \times 10^{-3}$ & $-1.81 \times 10^{-2}$ & $1.10 \times 10^{-2}$ & $2.12 \times 10^{-2}$ & $8.96 \times 10^{-3}$ & $1.31 \times 10^{-4}$ & $-2.63 \times 10^{-3}$ \\
\hline & ret. & $2.76 \times 10^{-3}$ & $2.74 \times 10^{-3}$ & $-8.48 \times 10^{-4}$ & $-2.02 \times 10^{-3}$ & $-1.93 \times 10^{-3}$ & $1.82 \times 10^{-4}$ & $1.75 \times 10^{-4}$ \\
\hline \multirow[t]{2}{*}{118} & mag. & $3.18 \times 10^{-2}$ & $-3.54 \times 10^{-3}$ & 0.1150 & 0.1796 & 0.0642 & $3.21 \times 10^{-2}$ & $1.27 \times 10^{-2}$ \\
\hline & ret. & $2.07 \times 10^{-3}$ & $2.15 \times 10^{-3}$ & -0.0143 & -0.0148 & -0.0128 & $-3.46 \times 10^{-3}$ & $-3.37 \times 10^{-3}$ \\
\hline$Z$ & & $5 f_{5 / 2}$ & $5 f_{7 / 2}$ & $6 s_{1 / 2}$ & $6 p_{1 / 2}$ & $6 p_{3 / 2}$ & $6 d_{3 / 2}$ & $6 d_{5 / 2}$ \\
\hline \multirow[t]{2}{*}{86} & mag. & & & $6.64 \times 10^{-4}$ & $1.70 \times 10^{-3}$ & $1.94 \times 10^{-4}$ & & \\
\hline & ret. & & & $4.01 \times 10^{-5}$ & $-9.73 \times 10^{-5}$ & $-9.84 \times 10^{-5}$ & & \\
\hline \multirow[t]{2}{*}{118} & mag. & $-1.20 \times 10^{-2}$ & $-1.94 \times 10^{-2}$ & $2.41 \times 10^{-2}$ & $3.85 \times 10^{-2}$ & $8.49 \times 10^{-3}$ & $-2.30 \times 10^{-5}$ & $-3.01 \times 10^{-3}$ \\
\hline & ret. & $2.52 \times 10^{-3}$ & $2.47 \times 10^{-3}$ & $-2.62 \times 10^{-3}$ & $-2.47 \times 10^{-3}$ & $-2.07 \times 10^{-3}$ & $2.14 \times 10^{-4}$ & $1.79 \times 10^{-4}$ \\
\hline$Z$ & & $7 s_{1 / 2}$ & $7 p_{1 / 2}$ & $7 p_{3 / 2}$ & & & & \\
\hline \multirow[t]{2}{*}{118} & mag. & $2.67 \times 10^{-3}$ & $4.60 \times 10^{-3}$ & $8.30 \times 10^{-5}$ & & & & \\
\hline & ret. & $-1.49 \times 10^{-4}$ & $-9.87 \times 10^{-5}$ & $-1.03 \times 10^{-4}$ & & & & \\
\hline
\end{tabular}

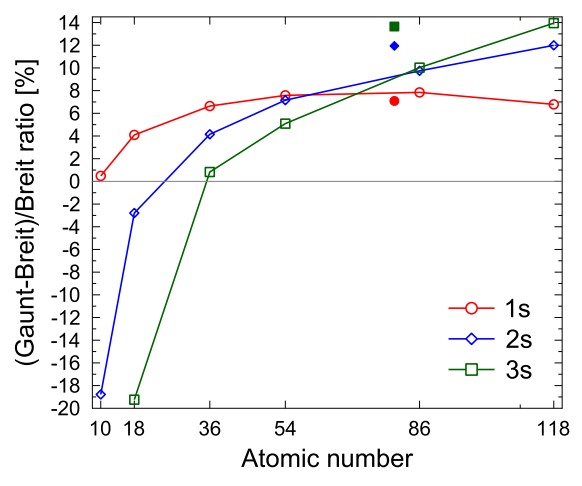

(a)

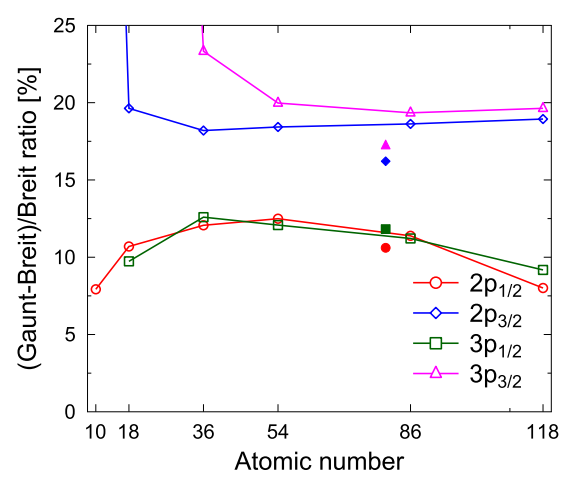

(b)

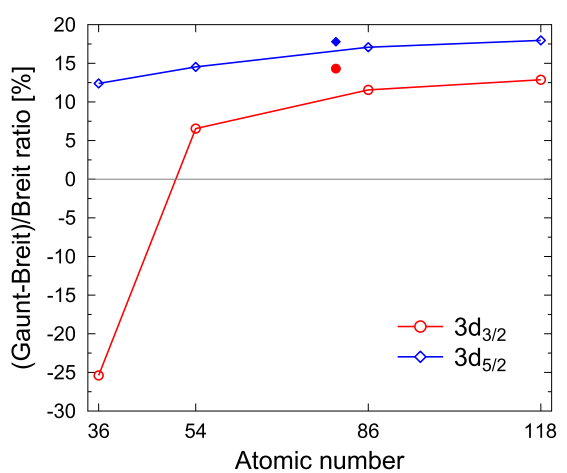

(c)

FIG. 3. Percentage difference of full Breit vs Gaunt contributions to the Dirac-Coulomb orbital energy for $n l(n \leq 3)$ subshells. Results from the work of Mann and Johnson ${ }^{24}$ are presented by filled markers. 


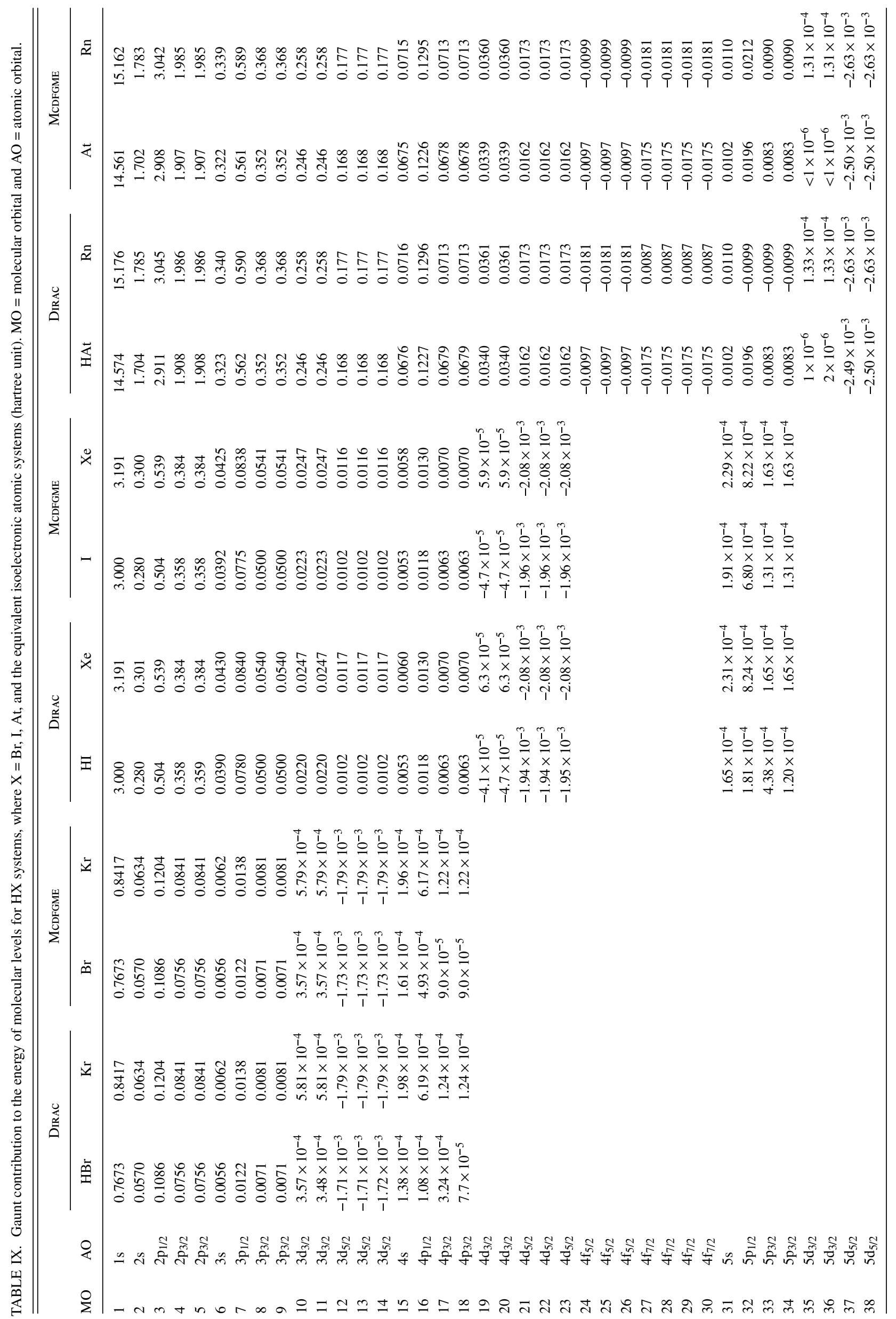


New insights appear. The $Z$-dependence is different for different shells even though the pattern of grow is similar in all cases. There is an increase of $b$ coefficient when $n$ increase, being its range of 3.627-5.473 for $n s$ subshells, of 4.263-5.216 for $n p_{1 / 2}$ subshells, and of 3.431-4.372 for $n p_{3 / 2}$ subshells. The pattern of the relationship among $n s_{1 / 2}, n p_{1 / 2}$, and $n p_{3 / 2}$ is such that (i) the a coefficient of $n p_{3 / 2}$ is one order of magnitude larger than the other two (which are of the same order of magnitude); (ii) for given $n$, the $b$ coefficients for $n p_{3 / 2}$ are smaller than those for $n s_{1 / 2}$ and $n p_{1 / 2}$ (see Table VI). This can be seen in Figs. $1(\mathrm{~b})-1(\mathrm{~d})$.

In both Table VII and Fig. 2, the Breit energy contributions to Dirac-Coulomb orbital energy ratios $\left(R^{B / D C}\right)$ for selected subshells are presented. It is clearly seen that the absolute value of the $R^{B / D C}$ ratio (as the Breit energy contribution has opposite sign than the Dirac-Coulomb orbital energy) increases when $Z$ increases. For given $n$, the following trend appears: $\operatorname{abs}\left(R_{n p_{1 / 2}}^{B / D C}\right)>\operatorname{abs}\left(R_{n p_{3 / 2}}^{B / D C}\right)>\operatorname{abs}\left(R_{n s_{1 / 2}}^{B / D C}\right)$. For $1 s$ subshell, the $R^{B / D C}$ ratio scales as $\sim Z^{1.1}$, what confirms the finding of Niskanen et al. ${ }^{26}$ for single and double 1 s electron ionization energies of noble gases.

\section{Gaunt vs retardation contribution}

We analyze here results of calculations performed with the MCDFGME code for Gaunt and full-Breit (i.e., a sum of Gaunt and retardation contributions) energy contributions for different subshells. In Table VIII, the magnetic (Gaunt) and retardation parts of Breit contributions to the Dirac-Coulomb orbital energy for $n l(n=1-3, l=0-2)$ orbitals of selected atoms have been collected. In Fig. 3, we show what kind of relationship both contributions to the orbital energies do follow. It is clearly seen that the full-Breit terms are smaller than the Gaunt ones, except the results for valence shells. That is because usually the Gaunt term and the retardation term have opposite signs. Considering only inner shells, the retardation term contributes to the full-Breit term about $10 \%$ for $n s$ and $n l_{l-1 / 2}$ (i.e., $n p_{1 / 2}$ and $n d_{3 / 2}$ ) subshells, and about $20 \%$ for $n l_{l+1 / 2}$ (i.e., $n p_{3 / 2}$ and $n d_{5 / 2}$ ) subshells. For $1 s$ shell, which is the most important one contributing to the total energy of any atomic system, the difference between Gaunt and full-Breit contributions is almost constant for high- $Z$ atoms and is about $7 \%-8 \%$. Our numbers are in agreement with the results of Lindroth et al., ${ }^{16}$ that the retardation part gives $5-10 \%$ contribution to the Breit interaction for the heavier elements. They are also in agreement with the finding of Indelicato,${ }^{51}$ that the retardation part has opposite sign and is about $2-10 \%$ of magnetic part for $1 s$ binding energy of Ne-like and Rn-like ions of superheavy elements $(Z=100-172)$. Mann and Johnson ${ }^{24}$ studied frozen-orbital binding energies (which are equivalent to orbital energies) of $\mathrm{Hg}$ and the values of Gaunt vs. full-Breit difference. Their findings are close to ours, though they are bigger in the case of $n s(n \geq 2)$ subshells. Recently, Niskanen et al. ${ }^{26}$ found that the Gaunt vs. full-Breit difference is about 3\%-7\% in the case of $1 s$ single ionization energies of noble gases $(\mathrm{Ne}$ to $\mathrm{Rn}$ ), and $5 \%-11 \%$ in the case of their $1 s$ double ionization energies. We found that for valence $n p_{3 / 2}$ subshells the Gaunt and retardation contributions are of comparable size. It is worth to note that for some outer (valence or near to valence) 
$s$ - or $d$-type orbitals the retardation part of Breit correction has the same sign as the magnetic part. This same finding has been found by Huang et $a .^{25}$ for relaxed-orbital binding energies.

\section{Gaunt contribution for molecular orbitals}

The Gaunt contributions to $\mathrm{Kr}, \mathrm{Xe}$, and $\mathrm{Rn}$ noble gases and its iso-electronic $\mathrm{HBr}, \mathrm{HI}$, and HAt diatomic molecules (calculated by using DiRAc code) are presented in Table IX. The atomic calculations for $\mathrm{Br}, \mathrm{I}, \mathrm{At}, \mathrm{Kr}, \mathrm{Xe}$, and $\mathrm{Rn}$, performed with the MCDFGME code, are also presented for comparison. Note that for $j>1 / 2$, the degeneration of $n l_{j}$ atomic levels is reduced in the HX molecule because of axial symmetry. The $Z$-dependence of Gaunt, as well as Breit, contribution to the orbital energy can be approximated by a power function, being the exponent about 4-5. For inner subshells, where molecular orbitals of $\mathrm{HX}(\mathrm{X}=\mathrm{Br}, \mathrm{I}, \mathrm{At})$ diatomic system are very similar to the atomic orbitals of the $\mathrm{X}$ atom, the Gaunt contribution to the energy of the $n l$ atomic orbital of noble gas is always larger than the Gaunt contribution to the energy of the corresponding molecular orbital of the HX molecule. For this case, the exponential Z-dependence of Gaunt/Breit contribution is clearly visible. For outer subshells, where molecular orbitals of the HX diatomic system are far from atomic orbitals of the $\mathrm{X}$ atom, the Gaunt contribution to the energy of the $n l$ atomic orbital of noble gas may be smaller or higher or may be of opposite sign than the Gaunt contribution to the energy of the corresponding molecular orbital of the HX molecule.

\section{CONCLUSIONS}

In this study, we investigated Breit effects (magnetic and retarded) on individual atomic and molecular orbital energies. Based on the findings presented above, a few main conclusions can be drawn.

(1) We can state that both frequency-dependent selfconsistent and perturbational approaches to calculate the Breit energy contribution provide accurate results. This was validated by their comparison with experiments on the $1 s$ ionization potential for Xe and $\mathrm{Rn}$. However, in the case of Rn $1 s$ ionization potential, the self-consistent approach to calculate the Breit energy contribution seems to be a little more accurate.

(2) The $Z$-dependence of Breit contribution to orbital energies has been evaluated by fitting Breit contributions to the orbital $n s$ and $n p$ energies by $a \times Z^{b}$ power function. The $a$ and $b$ coefficients may be used to estimate Breit effects on inner molecular orbitals. We observe some clean patterns: (i) for given $n, \varepsilon_{n p_{1 / 2}}^{B}>\varepsilon_{n p_{3 / 2}}^{B}$ and $\varepsilon_{n p_{1 / 2}}^{B}>\varepsilon_{n s}^{B}$; (ii) $\varepsilon_{n s}^{B}<\varepsilon_{n p_{3 / 2}}^{B}$ for $Z \lesssim 80-90(Z<91$ for $n=2$ and $Z<86$ for $n=3$ ) but opposite dependence for higher $Z$.

(3) We found that the retardation part contributes to the fullBreit term below $8 \%$ in the case of $1 s$ orbital energy but larger $(\simeq 20 \%$ or even more) for outer subshells.

(4) Comparison between the Gaunt term energy contribution per molecular level of HX systems (where
$\left.\mathrm{X}={ }_{35} \mathrm{Br},{ }_{53} \mathrm{I},{ }_{85} \mathrm{At}\right)$ and per atomic orbital of the equivalent isoelectronic atomic systems $\left({ }_{35} \mathrm{Kr},{ }_{54} \mathrm{Xe},{ }_{86} \mathrm{Rn}\right)$ shows that (i) for inner orbitals the Gaunt contributions to molecular systems are close to that of atomic systems, and (ii) for outer orbitals these contributions to molecular orbitals cannot be accurately reproduced by the corresponding atomic contributions.

For valence shells, the retardation term is a major part of the Breit interaction; therefore, there is a need for more study on Breit corrections to molecular level energies. We hope that our work will stimulate to implement the retardation term of the Breit interaction in leading molecular codes, such as the Dirac code.

\section{ACKNOWLEDGMENTS}

We gratefully acknowledge partial support from SGCyTUNNE and the Argentinian National Research Council for Science and Technology (CONICET, Grant No. PIP 11220090100654).

${ }^{1}$ P. Pyykkö, Chem. Rev. 112, 371 (2012).
${ }^{2}$ P. Indelicato, J. P. Santos, S. Boucard, an

${ }^{2}$ P. Indelicato, J. P. Santos, S. Boucard, and J.-P. Desclaux, Eur. Phys. J. D 45, 155 (2007).

${ }^{3}$ H. A. Bethe and E. E. Salpeter, "Quantum mechanics of one- and twoelectron systems," in Atoms I/Atome I (Springer Berlin Heidelberg, Berlin, Heidelberg, 1957), pp. 88-436.

${ }^{4}$ Y.-K. Kim, Phys. Rev. 154, 17 (1967).

${ }^{5}$ T. Kagawa, Phys. Rev. A 12, 2245 (1975).

${ }^{6}$ H. M. Quiney, I. P. Grant, and S. Wilson, J. Phys. B: At. Mol. Phys. 20, 1413 (1987)

${ }^{7}$ B. J. McKenzie, I. P. Grant, and P. H. Norrington, Comput. Phys. Commun. 21, 233 (1980).

${ }^{8}$ G. Breit, Phys. Rev. 34, 553 (1929).

${ }^{9}$ G. Breit, Phys. Rev. 36, 383 (1930).

${ }^{10}$ G. Breit, Phys. Rev. 39, 616 (1932).

${ }^{11}$ Y. Ishikawa, Chem. Phys. Lett. 166, 321 (1990).

${ }^{12}$ Y. Ishikawa, H. M. Quiney, and G. L. Malli, Phys. Rev. A 43, 3270 (1991).

${ }^{13}$ G. E. Brown and D. G. Ravenhall, Proc. R. Soc. A 208, 552 (1951).

${ }^{14}$ I. P. Grant, Proc. R. Soc. A 262, 555 (1961).

${ }^{15}$ H. M. Quiney, I. P. Grant, and S. Wilson, J. Phys. B: At., Mol. Opt. Phys. 23, L271 (1990).

${ }^{16}$ E. Lindroth, A.-M. Martensson-Pendrill, A. Ynnerman, and P. Oster, J. Phys. B: At., Mol. Opt. Phys. 22, 2447 (1989).

${ }^{17}$ M. H. Chen, B. Crasemann, and H. Mark, Phys. Rev. A 25, 391 (1982).

${ }^{18}$ A. M. Costa, M. C. Martins, J. P. Santos, P. Indelicato, and F. Parente, J. Phys. B: At., Mol. Opt. Phys. 40, 57 (2007).

${ }^{19}$ M. Pernpointner, J. Phys. B: At., Mol. Opt. Phys. 35, 383 (2002).

${ }^{20}$ M. Pernpointner, J. Phys. B: At., Mol. Opt. Phys. 38, 1955 (2005).

${ }^{21}$ H. M. Quiney, P. Barletta, G. Tarczay, A. G. Császár, O. L. Polyansky, and J. Tennyson, Chem. Phys. Lett. 344, 413 (2001).

${ }^{22}$ C. Thierfelder and P. Schwerdtfeger, Phys. Rev. A 82, 062503 (2010).

${ }^{23}$ C. T. Chantler, T. V. B. Nguyen, J. A. Lowe, and I. P. Grant, Phys. Rev. A 90, 062504 (2014).

${ }^{24}$ J. B. Mann and W. R. Johnson, Phys. Rev. A 4, 41 (1971).

${ }^{25}$ K.-N. Huang, M. Aoyagi, M. H. Chen, B. Crasemann, and H. Mark, At. Data Nucl. Data Tables 18, 243 (1976).

${ }^{26}$ J. Niskanen, P. Norman, H. Aksela, and H. Ågren, J. Chem. Phys. 135, 054310 (2011).

${ }^{27}$ F. A. Parpia, A. K. Mohanty, and E. Clementi, J. Phys. B: At., Mol. Opt. Phys. 25, 1 (1992).

${ }^{28}$ A. Canal Neto, P. Librelon, and F. Jorge, Chem. Phys. Lett. 326, 501 (2000).

${ }^{29}$ O. Visser, L. Visscher, P. J. C. Aerts, and W. C. Nieuwpoort, Theor. Chim. Acta 81, 405 (1992).

${ }^{30}$ G. L. Malli and J. Styszyński, J. Chem. Phys. 104, 1012 (1996).

${ }^{31}$ J. Styszyński, X. Cao, G. L. Malli, and L. Visscher, J. Comput. Chem. 18, 601 (1997).

${ }^{32}$ I. P. Grant, in Methods in Computational Chemistry (Springer US, Boston, MA, 1988), Vol. 2, pp. 1-71. 
${ }^{33}$ I. P. Grant, Relativistic Quantum Theory of Atoms and Molecules, Volume 40 of Springer Series on Atomic, Optical, and Plasma Physics (Springer, New York, NY, 2007).

${ }^{34}$ I. P. Grant, J. Phys. B: At., Mol. Opt. Phys. 43, 074033 (2010).

${ }^{35}$ I. P. Grant, Adv. Phys. 19, 747 (1970).

${ }^{36}$ O. Gorceix, P. Indelicato, and J.-P. Desclaux, J. Phys. B: At. Mol. Phys. 20, 639 (1987)

${ }^{37}$ P. Indelicato, O. Gorceix, and J.-P. Desclaux, J. Phys. B: At. Mol. Phys. 20, 651 (1987).

${ }^{38}$ I. Lindgren, Relativistic Many-Body Theory: A New Field-Theoretical Approach, Volume 63 of Springer Series on Atomic, Optical, and Plasma Physics (Springer-Verlag, New York, 2011).

${ }^{39}$ C. Møller, Ann. Phys. 406, 531 (1932).

${ }^{40}$ J. A. Gaunt, Proc. R. Soc. A 122, 513 (1929).

${ }^{41}$ P. Indelicato, S. Boucard, and E. Lindroth, Eur. Phys. J. D 3, 29 (1998).

${ }^{42}$ R. D. Deslattes, E. G. Kassler, P. Indelicato, L. De Billy, E. Lindroth, and J. Anton, Rev. Mod. Phys. 75, 35 (2003).

${ }^{43}$ M. Barysz and Ł. Syrocki, Mol. Phys. 112, 583 (2014).

${ }^{44}$ J. A. Bearden and A. F. Burr, Rev. Mod. Phys. 39, 125 (1967).

${ }^{45}$ M. Deutsch, G. Brill, and P. Kizler, Phys. Rev. A 43, 2591 (1991).

${ }^{46}$ O. Gorceix and P. Indelicato, Phys. Rev. A 37, 1087 (1988).

${ }^{47}$ J. Sucher, J. Phys. B: At., Mol. Opt. Phys. 21, L585 (1988).

${ }^{48}$ E. Lindroth and A.-M. Mårtensson-Pendrill, Phys. Rev. A 39, 3794 (1989).

${ }^{49}$ I. Lindgren, J. Phys. B: At., Mol. Opt. Phys. 23, 1085 (1990).

${ }^{50}$ I. P. Grant and N. C. Pyper, J. Phys. B: At. Mol. Phys. 9, 761 (1976).
${ }^{51}$ P. Indelicato, J. Phys. B: At. Mol. Phys. 19, 1719 (1986).

${ }^{52}$ N. Beatham, I. P. Grant, B. J. McKenzie, and S. J. Rose, Phys. Scr. 21, 423 (1980).

${ }^{53}$ P. Indelicato and J.-P. Desclaux, "MCDFGME, a multiconfiguration Dirac-Fock and General Matrix Elements program (release 2005)," http://dirac.spectro.jussieu.fr/mcdf.

${ }^{54}$ J.-P. Desclaux, Comput. Phys. Commun. 9, 31 (1975).

${ }^{55}$ J.-P. Desclaux, Comput. Phys. Commun. 35, C288 (1984).

${ }^{56}$ K. G. Dyall, I. P. Grant, C. Johnson, F. A. Parpia, and E. Plummer, Comput. Phys. Commun. 55, 425 (1989).

${ }^{57}$ P. Jönsson, X. He, C. Froese Fischer, and I. P. Grant, Comput. Phys. Commun. 177, 597 (2007).

${ }^{58}$ P. Jönsson, G. Gaigalas, J. Bieroń, C. Froese Fischer, and I. P. Grant, Comput. Phys. Commun. 184, 2197 (2013).

${ }^{59}$ DIRAC, a relativistic ab initio electronic structure program, Release DIRAC13, written by L. Visscher, H. J. Aa. Jensen, R. Bast, and T. Saue, with contributions from V. Bakken, K. G. Dyall, S. Dubillard, U. Ekström, E. Eliav, T. Enevoldsen, E. Faßhauer, T. Fleig, O. Fossgaard, A. S. P. Gomes, T. Helgaker, J. K. Lærdahl, Y. S. Lee, J. Henriksson, M. Iliaš, Ch. R. Jacob, S. Knecht, S. Komorovský, O. Kullie, C. V. Larsen, H. S. Nataraj, P. Norman, G. Olejniczak, J. Olsen, Y. C. Park, J. K. Pedersen, M. Pernpointner, K. Ruud, P. Sałek, B. Schimmelpfennig, J. Sikkema, A. J. Thorvaldsen, J. Thyssen, J. van Stralen, S. Villaume, O. Visser, T. Winther, and S. Yamamoto, see http://www.diracprogram.org, 2013.

${ }^{60}$ C. A. Giménez, K. Kozioł, and G. A. Aucar, Phys. Rev. A 93, 032504 (2016).

${ }^{61}$ SciDAVis, http://scidavis.sourceforge.net. 